\title{
Capacidade estatal e desempenho na percepção dos burocratas brasileiros: desenvolvimento e validação de um modelo de equações estruturais
}

\author{
ALeXANDre de Ávila Gomide ${ }^{1}$ \\ RAPHAEL AMORIM MACHADO ${ }^{1}$ \\ Pedro Melo Albuquerque ${ }^{2}$
}

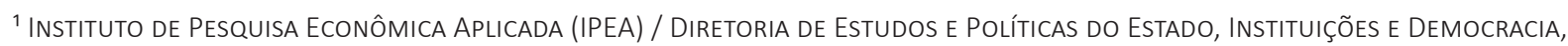 \\ BRASÍLIA - DF, BRASIL \\ 2 Universidade de Brasília (UnB) / Programa de Pós-GraduaÇÃo em AdMinistração, Laboratório de Aprendizado de MÁQuina EM \\ FINANÇAS E ORGANIZAÇÕES, BRASÍLIA - DF, BRASIL
}

\begin{abstract}
Resumo
Este artigo especifica e valida um modelo derivado de abordagens teóricas na literatura para mensurar as capacidades do Estado, especificamente do governo federal brasileiro. Dados coletados por Survey foram analisados usando a técnica de modelagem de equações estruturais (MEE). Os achados indicam que as características weberianas da burocracia ainda são uma referência útil para estudos sobre a capacidade estatal, uma vez que o nível de profissionalização e de habilidades dos burocratas apresentaram efeito positivo e estatisticamente significativo sobre o desempenho percebido do Estado. No que diz respeito à autonomia burocrática, os achados indicam que seu efeito no desempenho do Estado é indireto, mediado pela profissionalização. Ao contrário das previsões teóricas, não encontramos efeitos diretos significativos entre os relacionamentos da burocracia com atores não estatais e desempenho do Estado nem entre este e a dotação de recursos organizacionais. O artigo contribui para a literatura ao utilizar dados obtidos diretamente dos burocratas, ao desenvolver e validar um modelo replicável que relaciona as diferentes dimensões do conceito de capacidades estatais e ao utilizar a MEE para estimar os efeitos das dimensões do conceito sobre os resultados da ação estatal.
\end{abstract}

Palavras-chave: Burocracia. Autonomia. Capacidade estatal. Modelagem de equações estruturais. Desempenho do Estado.

State capacity and performance from the perception of Brazilian bureaucrats: development and validation of a structural equation model

\begin{abstract}
The study specified and validated a model derived from theoretical approaches in the literature to measure state capacities. Survey data were analyzed using structural equation modeling (SEM). The findings indicate that bureaucracy's Weberian features are still a valuable reference for state capacity studies since bureaucrats' professionalization and skills presented a positive and statistically significant effect on the state's perceived performance. Concerning bureaucratic autonomy, the findings indicate that its effect on state performance is indirect, mediated by professionalization. Unlike theoretical predictions, we found no significant direct effects of bureaucracy relationships with non-state actors and of organizational resources for state performance. The article contributes to the literature by using data obtained directly from bureaucrats, developing and validating a replicable model that relates the different dimensions of the concept, and using SEM to estimate the effects of each of the concept's dimensions of state capacity on the outcomes of State action.
\end{abstract}

Keywords: Bureaucracy. Autonomy. State capacity. Structural equation modeling. State performance.

Capacidad estatal y desempeño en la percepción de los burócratas brasileños: desarrollo y validación de un modelo de ecuaciones estructurales

\section{Resumen}

Este artículo especifica y valida un modelo derivado de enfoques teóricos en la literatura para medir la capacidad estatal, específicamente del Gobierno Federal brasileño. Se analizaron los datos recopilados mediante encuestas utilizando la técnica de modelado de ecuaciones estructurales (MEE). Los resultados indican que los criterios weberianos de burocracia siguen siendo una referencia útil para los estudios sobre la capacidad del Estado, ya que la profesionalización, las habilidades y la autonomía individual de los burócratas tienen un efecto positivo y estadísticamente significativo sobre el desempeño percibido del Estado. Sin embargo, a diferencia de las predicciones teóricas, no encontramos efectos directos significativos de las relaciones de la burocracia con los actores no estatales, así como de los recursos organizacionales para el desempeño percibido de los Estados. El artículo contribuye a la literatura al desarrollar y validar un modelo replicable que relaciona y mide diferentes dimensiones del concepto de capacidad estatal y también al utilizar la técnica MEE para estimar los efectos de cada una de las dimensiones del concepto sobre los resultados de la acción estatal.

Palabras clave: Burocracia. Autonomía. Capacidad estatal. Modelo de ecuaciones estructurales. Desempeño del Estado. 


\section{INTRODUÇÃO}

O ressurgimento do interesse pelo conceito de capacidade estatal na literatura indica o reconhecimento de sua importância analítica para compreender a atuação do Estado e explicar uma variedade de resultados de políticas públicas. Assim, podemos observar esforços para a definição, a operacionalização e a mensuração do conceito (Cingolani, 2018; Hanson \& Sigman, 2019; Savoia \& Sen, 2012). Publicações recentes, como a de Centeno, Kohli e Yashar (2017), discutem o conceito de capacidade estatal no centro do debate teórico para a perspectiva do desenvolvimento e do funcionamento do Estado moderno, indicando a necessidade de acionamento político das capacidades existentes. Observamos também desenvolvimentos de autores tanto das áreas de administração e políticas públicas (Wu, Ramesh \& Howlett, 2015) quanto da ciência política (Fukuyama, 2013) em operacionalizar e mensurar o conceito. Tais desenvolvimentos vêm a se somar às elaborações seminais dos neoinstitucionalistas da escola histórica (Evans, 1995) quando explicaram a eficácia das políticas de transformação estrutural implementadas pelos Estados desenvolvimentistas do Leste Asiático.

No Brasil, os trabalhos publicados apontam a atualidade do conceito no debate acerca da administração pública brasileira. Pesquisas coordenadas por Gomide e Boschi (2016) se utilizaram do conceito para analisar o papel do Estado no desenvolvimento. De igual modo, Pires e Gomide (2016) avaliaram o efeito dos arranjos institucionais sobre as capacidades estatais e os resultados de políticas públicas, ao passo que Bersh, Praça e Taylor (2017) utilizaram o conceito para analisar o efeito da politização da burocracia das agências federais na corrupção. Em nível subnacional, podemos citar os trabalhos de Marenco, Strohschoen e Joner (2017), que examinam em que medida os municípios com burocracias profissionalizadas foram mais capazes de aproveitar a transferência de autoridade política e administrativa para tributar e implementar políticas públicas, bem como os de Grin, Nascimento, Abrucio e Fernandes (2018), que analisam a relação entre gestão pública e desempenho fiscal municipal considerando 4 dimensões das capacidades locais: administrativa, técnica, institucional e política. Ainda no nível subnacional, Satyro e Cunha (2018) avaliaram a capacidade do governo federal de construir uma burocracia da assistência social nos municípios brasileiros.

Por se tratar de um conceito não observável e multidimensional, autores como Souza e Fontanelli (2020), além Gomide, Machado e Pereira (2019), discutiram a importância de sua decomposição em atributos e da reunião de indicadores para mensurá-los. Em trabalho pioneiro no Brasil, Souza (2016) calculou um índice de qualidade da burocracia brasileira (IQB) nas agências do governo federal responsáveis pelas seguintes áreas de política pública: meio ambiente, infraestrutura, inovação e industrial.

Neste trabalho, objetivamos desenvolver um modelo de mensuração de capacidades estatais para o governo federal brasileiro informado por diferentes abordagens teóricas e verificá-lo empiricamente com base em dados empíricos obtidos diretamente com burocratas da administração federal brasileira, por meio de Survey. Para isso, utilizaremos a modelagem de equações estruturais (MEE), uma técnica de análise de dados multivariada adequada para testar teorias ou relações de associação entre variáveis observáveis e latentes (não observadas). De tal modo, buscamos avaliar, com base em evidências, a aderência empírica de diferentes abordagens teóricas, confirmando se as dimensões constitutivas do conceito informadas por tais abordagens são empiricamente corroboradas, se existem relações entre elas e, por fim, se apresentam efeitos ou relações significativas sobre o resultado da ação estatal.

O artigo contribui para a literatura sobre o conceito de 3 modos: empiricamente, ao utilizar dados obtidos diretamente com os burocratas, por meio de Survey; teoricamente, ao desenvolver e validar um modelo replicável que relaciona as diferentes dimensões do conceito de capacidade estatal; e metodologicamente, ao utilizar a MEE para estimar os efeitos de cada uma das dimensões do conceito sobre os resultados percebidos da ação do Estado.

Na próxima seção são discutidas as abordagens teóricas do conceito de capacidade estatal que embasam o modelo teórico testado empiricamente. A seção 3 apresenta o Survey que foi aplicado para a coleta de dados. Por sua vez, a seção 4 explica como as dimensões constitutivas do conceito, informadas pelas abordagens apresentadas, foram operacionalizadas e mensuradas. A seção 5 apresenta o modelo de equações estruturais que foi hipotetizado, seguida pela apresentação dos resultados, na seção 6. Por fim, a seção 7 conclui o artigo, destacando os principais achados e as limitações do trabalho. 


\section{CAPACIDADE ESTATAL: DIFERENTES ABORDAGENS E DIMENSÕES CONSTITUTIVAS}

Existem várias acepções sobre o conceito de capacidade estatal, mas sua definição mais ampla está relacionada à habilidade do Estado para alcançar os próprios objetivos, ou seja, implementar suas decisões, sobretudo a despeito da oposição de grupos de interesse e classes sociais (Skocpol, 1985). Nessa perspectiva, o conceito é associado a características organizacionais das burocracias, como sua competência e autonomia em relação aos grupos de interesse. Nesse registro, a ideia é signatária de um elemento fundante, que está associado ao tipo ideal weberiano de burocracia: profissional e baseada no mérito. Para além dessas qualidades, a burocracia deve ser dotada dos recursos materiais necessários para que sua atuação seja eficaz.

A capacidade estatal não pode ser observada diretamente, pois se trata de um construto. Assim, como qualquer noção social, ela precisa ser definida em bases teóricas e mensurada empiricamente. Para isso, é preciso ser operacionalizada em seus atributos ou dimensões constitutivas (Beach \& Pedersen, 2016). Para Goertz (2006), um conteúdo pode ser decomposto em três níveis: básico (ontológico ou teórico), constitutivo (secundário ou dimensional) e indicativo (realista ou observável). O primeiro nível foca no que é cognitivamente central do fenômeno, o segundo identifica os atributos ou as dimensões que desempenham papel nas explicações e seus mecanismos causais, ao passo que o terceiro operacionaliza os atributos mobilizados no nível anterior por meio de indicadores observáveis. A Figura 1, abaixo, exemplifica a explicação.

Figura 1

Exemplo de conceptualização em 3 níveis

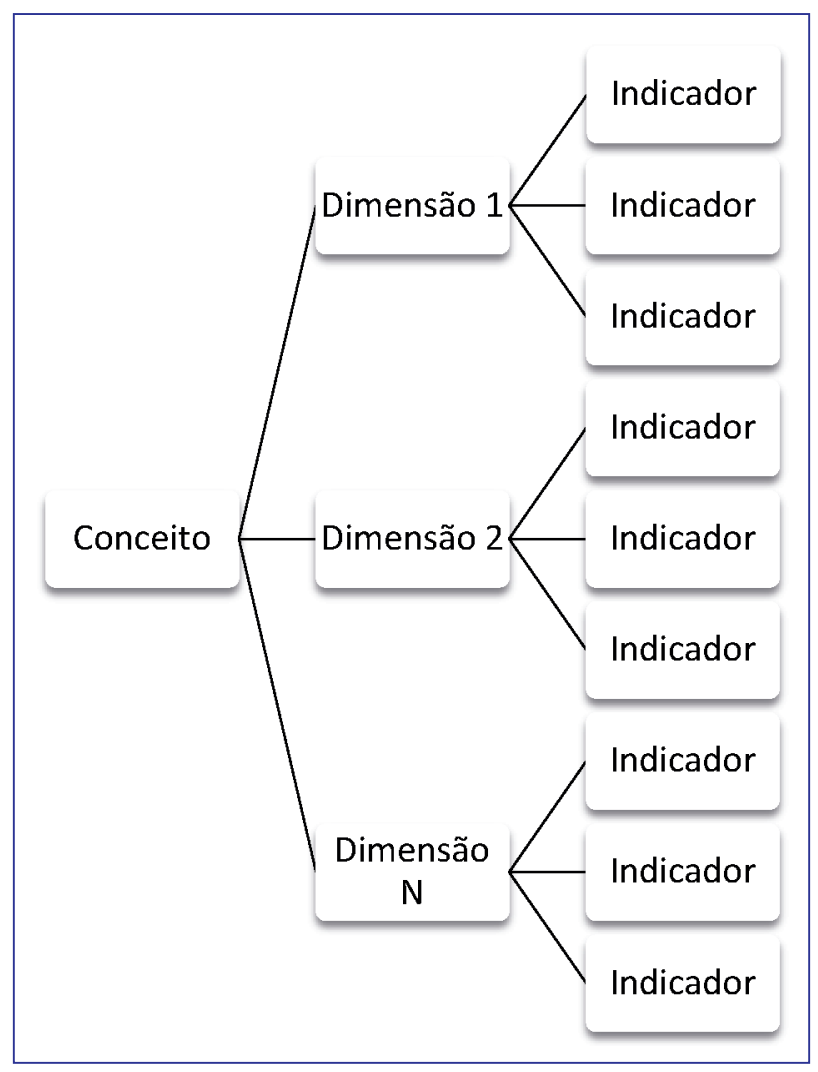

Fonte: Elaborada pelos autores.

São várias as abordagens teóricas que informam a definição e a operacionalização do conceito de capacidade estatal (Cingolani, 2013). Tendo em vista o objetivo deste trabalho, serão discutidas algumas delas com o objetivo de explorar suas dimensões constitutivas. A associação da noção de capacidade estatal às competências e aos recursos da burocracia pública foi o critério para a seleção das abordagens revisadas nesta seção. Na seguinte, tais dimensões serão operacionalizadas por meio de indicadores ou variáveis observáveis. 
Os neoinstitucionalistas da escola histórica associam a capacidade do Estado ao tipo ideal weberiano de burocracia (Evans, 1995). Para Weber, a burocracia é o meio mais eficiente pelo qual o Estado pode ser organizado. Assim, a organização burocrática é associada ao tipo racional-legal de dominação, sendo este uma condição necessária ao desenvolvimento do capitalismo. Características do tipo ideal weberiano de burocracia, como recrutamento e promoções baseadas no mérito e na estabilidade da carreira, criariam um ethos e um esprit de corps à instituição, dotando-a de maior coesão e coerência na ação, além de inflá-la de tentativas de captura pelos atores privados (Evans \& Rauch, 1999).

A autonomia da burocracia em relação às classes sociais e aos grupos de interesse é central na explicação de Evans (1995) sobre a capacidade dos Estados desenvolvimentistas do Leste Asiático. Contudo, ele também argumenta que as relações entre Estado e sociedade, ou dos burocratas com atores privados, são igualmente cruciais para a efetividade do Estado, pois elas fornecem as informações necessárias para que os burocratas formulem políticas consistentes e garantem a eficácia de sua implementação por uma densa rede de conexões (Evans, 1995, 2010). Assim, o conceito de "autonomia inserida" vem a conciliar tais perspectivas, ou seja, da necessidade de uma burocracia profissional e autônoma, mas ao mesmo tempo conectada ou inserida no meio social circundante.

Mais recentemente, Fukuyama (2013), ao discutir o conceito de governança, ou da capacidade do governo de fazer e aplicar leis e entregar bens e serviços públicos à sociedade, dialoga diretamente com a abordagem weberiana de burocracia. Na sua conceptualização, o autor privilegia a autonomia que os burocratas profissionais (agentes) detêm para implementar as ordens dos políticos (principais). Para Fukuyama, profissionalização é o recrutamento e a ascensão na carreira burocrática mediante critérios meritocráticos. De acordo com o autor, a autonomia não significa que os burocratas devam estabelecer os próprios objetivos. Assim, os políticos devem determinar os objetivos, mas não de que modo os implementar. $O$ inverso da autonomia seria a subordinação completa dos burocratas aos políticos ou o microgerenciamento das suas ações no momento da implementação das políticas públicas. Como uma burocracia baseada no mérito é necessária para que exista tal autonomia, Fukuyama decompõe o conceito de governança em 2 dimensões: profissionalização e autonomia individual.

Cabe aqui uma distinção entre a acepção da autonomia burocrática de Peter Evans e a de Francis Fukuyama. Para o primeiro, a autonomia (ou insulamento burocrático) seria em relação aos interesses dos grupos de interesse e classes econômicas, enquanto para o segundo a autonomia (ou discricionariedade no nível operacional) seria em relação aos procedimentos internos para implementação das ações definidas na esfera política. Essa distinção também serve para perspectiva de autonomia enfatizada por Carpenter (2001), cujo foco é a autonomia das organizações estatais, e não a autonomia individual dos servidores públicos.

Por fim, mas não menos importante, Wu et al. $(2015,2018)$ trabalham o conceito de policy capacity, ou capacidade para políticas públicas, em diferente nível de abstração, por meio de uma estrutura conceitual baseada nas habilidades e nos recursos considerados necessários ao policymaking. Os autores destacam que as abordagens acerca do conceito de capacidade estatal são voltadas para o nível macro - por exemplo, para comparar um conjunto de Estados. Por isso, diferentemente, eles centram sua abordagem nas questões concernentes à estrutura interna às burocracias públicas nos níveis individuais, organizacionais e sistêmico. Dessa maneira, operacionalizam o conceito dividindo as dimensões relativas a habilidades e recursos em 3 tipos: analítica, operacional e política - a relação entre os níveis analíticos e os tipos de habilidades e recursos resultam numa matriz $3 \times 3$, com 9 grupos de indicadores. No nível individual, a capacidade pode ser medida pelas habilidades técnicas, gerenciais e políticas dos burocratas, enquanto no nível organizacional, ela pode ser medida pela disponibilidade de recursos humanos, financeiros e de infraestrutura de informações necessários para uma agência desempenhar adequadamente suas funções. Por fim, no nível sistêmico, destaca-se o apoio de que um órgão público desfruta entre os políticos e a sociedade em geral como chave para a capacidade do Estado de produzir políticas públicas.

O Quadro 1 faz uma síntese das dimensões constitutivas do conceito com base em abordagens teóricas revisadas nesta seção. Tendo em vista o foco deste trabalho na burocracia estatal, privilegiaremos os níveis individuais e organizacionais da abordagem de Wu et al. $(2015,2018)$. 


\section{Quadro 1}

Abordagens sobre capacidade estatal

\begin{tabular}{|c|c|c|}
\hline Conceito & Dimensões constitutivas & Referências teóricas \\
\hline Autonomia inserida & "Weberianidade" & Evans (1995) \\
\hline Governança & Relacionamento com atores privados & Fukuyama (2013) \\
\hline Policy capacity & Autonomia burocrática & Wu et al. (2015) \\
\hline
\end{tabular}

Fonte: Elaborado pelos autores.

As 3 abordagens revisadas nesta seção não são rivais, e sim complementares. Enquanto os conceitos de "autonomia inserida" e de "governança" adotam um nível mais elevado de abstração, o de "policy capacity" adota uma abordagem de nível organizacional e individual. A complementaridade entre os níveis de abstração das 3 abordagens é compatível com o instrumento de mensuração discutido a seguir.

\section{O SURVEY COM A BUROCRACIA FEDERAL BRASILEIRA}

Os dados usados para análise foram coletados de um questionário aplicado pelo Instituto de Pesquisa Econômica e Aplicada (Ipea) aos servidores da administração pública federal brasileira, entre maio e julho de 2018 (Pereira et al., 2019), elaborado em parceria com a equipe do Projeto Governança, conduzido pelo Center on Democracy, Development and The Rule of Law (CDDRL), da Universidade de Stanford, coordenado por Francis Fukuyama. O questionário teve como base o Federal Employee Viewpoint Survey (FEVS), que busca medir as percepções dos burocratas federais estadunidenses acerca de características e condições de trabalho presentes em suas respectivas organizações. Algumas questões derivadas das abordagens teóricas utilizadas nessa pesquisa compuseram o questionário, como as utilizadas por Ramesh, Howlett e Saguin (2016) a fim de mensurar as policy capacities de burocracias públicas, além de algumas sugeridas pelos pesquisadores do Ipea para as especificidades do caso brasileiro. O questionário completo, assim como estatísticas descritivas dos dados coletados, se encontra em Pereira et al. (2019).

O universo amostral da pesquisa foi composto de servidores públicos federais brasileiros com vínculo permanente e postos comissionados, tanto de órgãos da administração direta quanto da administração indireta, como autarquias, fundações e agências reguladoras. Foram excluídos da amostra bancos e empresas públicas, além dos chamados burocratas de linha de frente, como policiais, médicos, enfermeiros e professores. A exclusão destes teve o objetivo de evitar distorções na amostra em virtude do número desses servidores em relação às demais carreiras de Estado. Além disso, buscamos focar nossa análise em burocratas com funções de formulação e avaliação de políticas públicas, fornecendo-nos, assim, um quadro mais preciso acerca das dotações de competências, recursos e relacionamentos no interior da administração pública federal.

As camadas do universo amostral foram delimitadas com base em 3 critérios: cargos e carreiras, cargo comissionado e modelo jurídico-organizacional do órgão no qual o servidor está lotado. O primeiro se subdividiu em 3: funcionários com vínculo estável com a administração pública, funcionários comissionados e outros, abarcando terceirizados, contratos temporários, consultores etc. O segundo também se subdividiu em 3: funcionários com função comissionada, portanto, necessariamente, estáveis; funcionários investidos em cargos de livre provimento - direção e assessoramento superior (DAS) ou equivalente -, tendo ou não vínculo estável com a administração pública; e outros, que não se enquadram em nenhum dos 2 critérios mencionados antes. O último critério, modelo jurídico organizacional, subdividiu a administração pública federal em 3 modelos: administração direta - que abarca a presidência da República e os ministérios -, autarquias ou fundações públicas de direito público e agências reguladoras - que são autarquias em regime especial. O recorte possibilitou ampla representatividade aos setores componentes da estrutura administrativa brasileira e contou com uma população amostral de 3.200 servidores, de um universo de 263.498. Foram enviados 14.704 questionários, e a taxa de respostas ficou em $21 \%$. Detalhes sobre as fórmulas de amostragem, a composição das camadas dos servidores e os órgãos selecionados para comporem a amostra também podem ser encontrados em Pereira et al. (2019). 
Comparativamente, a qualidade da burocracia federal brasileira é bem avaliada em rankings internacionais, superando as outras nações latino-americanas numa variedade de índices, como Economist Intelligence Unit, da revista The Economist; Political Risk Services, do Fundo Monetário Internacional (FMI); Worldwide Governance Indicators, do Banco Mundial; e Quality of Government, da Universidade de Gotemburgo. A burocracia federal ganhou seus primeiros contornos de profissionalização já na década de 1930, combinando seleção meritocrática para importantes carreiras de Estado, patronagem em carreiras da administração indireta e elementos de insulamento burocrático de agências e órgãos centrais para o desenvolvimento (Sikkink, 1991). Após a redemocratização, a Constituição de 1988 exigiu a seleção por concurso como o principal meio de ingresso no serviço público federal (Souza, 2016), fator que explica os elevados índices de profissionalização observados hoje em dia.

\section{OPERACIONALIZAÇÃO E MENSURAÇÃO DAS DIMENSÕES CONSTITUTIVAS}

Nesta seção, explicamos como as dimensões constitutivas do conceito informadas pelas abordagens teóricas revisadas na seção 2 foram operacionalizadas para mensuração. Tendo em vista que o trabalho objetiva também verificar os efeitos das dimensões constitutivas sobre o desempenho estatal, apresentamos uma discussão acerca da mensuração dos resultados da ação das burocracias. Com isso, distingue-se a capacidade estatal dos seus resultados, evitando o problema do raciocínio tautológico na aplicação do conceito.

\section{A capacidade estatal como um construto}

Como discutido, a capacidade estatal é um construto formado por dimensões observáveis apenas indiretamente por meio de um conjunto de indicadores. Assim, com base nas abordagens teóricas revisadas, foram selecionadas perguntas do questionário para construir e mensurar as dimensões constitutivas do conceito (vide Quadro 2). As respostas às questões obedeceram à escala de Likert, variando de 1 a 5, sendo 1 total discordância com relação à afirmação e 5, total concordância. As perguntas selecionadas refletem as abordagens teóricas revisadas na seção 2 , referindo-se tanto a questões mais gerais de governo quanto a características organizacionais e individuais dos burocratas.

\section{Quadro 2}

\section{Dimensões e indicadores}

\begin{tabular}{|c|c|}
\hline Dimensão & Indicadores (Questões) \\
\hline Profissionalização & $\begin{array}{l}\text { QB1 - Minha unidade de trabalho é capaz de recrutar pessoas com as habilidades corretas. } \\
\text { QB2 - Promoções na carreira em minha unidade de trabalho são baseadas no mérito. } \\
\text { QB5 - Competência técnica é importante para nomeação a um cargo comissionado. }\end{array}$ \\
\hline Autonomia & $\begin{array}{l}\text { QC1 - Minha percepção é que o governo utiliza ao máximo minhas habilidades. } \\
\text { QC2 - Em meu atual cargo, sinto-me encorajado a trazer novos e melhores modos de trabalho. } \\
\text { QC3 - Tenho condições para tomar decisões próprias na realização do meu trabalho. }\end{array}$ \\
\hline Recursos & $\begin{array}{l}\text { Os itens a seguir são obstáculos ao bom desempenho da sua unidade de trabalho: } \\
\text { QE1 - Recursos humanos; } \\
\text { QE2 - Recursos orçamentários; } \\
\text { QE3 - Recursos tecnológicos. }\end{array}$ \\
\hline Habilidades & $\begin{array}{l}\text { Os servidores de sua organização têm as habilidades descritas a seguir: } \\
\text { QG1 - Conhecimentos das políticas públicas do setor de atuação; } \\
\text { QG3 - Habilidades para analisar políticas públicas; } \\
\text { QG7 - Habilidades de comunicação. }\end{array}$ \\
\hline $\begin{array}{l}\text { Relacionamento } \\
\text { com atores não } \\
\text { estatais }\end{array}$ & $\begin{array}{l}\text { Nos últimos } 12 \text { meses, com que frequência você interagiu com: } \\
\text { QF8 - Empresas privadas; } \\
\text { QF10 - Organizações da sociedade civil. }\end{array}$ \\
\hline
\end{tabular}

Fonte: Elaborado pelos autores. 
Com base em Fukuyama (2013) e Boittin, Distelhorst e Fukuyama (2016), as formas de recrutamento, promoções na carreira e indicações a cargos comissionados foram selecionadas como indicadores da dimensão de profissionalização burocrática. A operacionalização da dimensão da autonomia também seguiu a abordagem de Fukuyama e adotada por Boittin et al. (2016) com indicadores sobre o aproveitamento das habilidades individuais dos servidores, o encorajamento para eles trazerem novos métodos de trabalho e o grau de discricionariedade concedida para que possam tomar decisões na realização dos seus trabalhos. A esse respeito, os indicadores selecionados buscam indícios de que rotinas de trabalho excessivamente rígidas limitam o desempenho das burocracias públicas, desencorajando o uso de conhecimento profissional, criatividade e interações humanas na consecução das funções da burocracia.

Em conformidade com Ramesh et al. (2016), foram selecionadas perguntas no questionário aplicado para operacionalizar e mensurar as dimensões respectivas aos estoques de recursos e as habilidades que detêm os burocratas, conforme a conceptualização de policy capacity de Wu et al. (2015). Assim, as repostas sobre a disponibilidade de recursos humanos, orçamentários e tecnológicos nas organizações às quais pertencem os respondentes e a percepção dos burocratas sobre os conhecimentos que detêm seus pares sobre as políticas do setor de atuação, as habilidades para analisar as políticas públicas e de comunicação interpessoal foram considerados indicadores das variáveis latentes "recursos" e "habilidades", respectivamente.

Por fim, as perguntas relacionadas à frequência da interação dos burocratas com empresas privadas e organizações da sociedade civil foram selecionadas para operacionalizar e mensurar o construto sobre o relacionamento entre Estado e sociedade. Ainda que tal frequência não necessariamente esclareça o conteúdo e o sentido de tais relações, podendo se referir a distintos níveis - formal ou informal, direta ou por canais institucionais -, estas foram consideradas proxies imperfeitas da referida dimensão.

\section{O desempenho da ação estatal}

A questão do desempenho da ação do Estado e sua mensuração é muito discutida pela literatura (Andrews, Boyne \& Walker, 2011; Fernandez \& Moldogaziev, 2013, 2015; March \& Sutton, 1997). Diante do desafio de encontrar medidas-padrão para organizações pertencentes a diferentes setores de políticas públicas, optamos por dados provenientes das perguntas do questionário por serem comuns a todos, apesar das limitações dessa alternativa. Entre elas, destacam-se a subjetividade da interpretação das perguntas pelos respondentes e o possível viés causado pela simpatia que o respondente tem em relação à organização à qual pertence (o chamado "efeito halo").

Fernandez e Moldogaziev (2013) observaram, com os dados da burocracia estadunidense, que as medidas de percepção do desempenho organizacional se correlacionaram positiva e significativamente com as medidas objetivas. Ademais, a análise de Andrews et al. (2011) sobre um amplo conjunto de estudos acerca do desempenho estatal não encontrou evidências de que avaliações baseadas nas respostas dos próprios servidores públicos tenderiam a inflar avaliações do impacto positivo de seus esforços. Assim, o pressuposto de que medidas perceptivas internas, como questões de Survey, possam servir como substitutos de critérios externos objetivos do desempenho organizacional foi adotado para efeitos deste trabalho.

Foram escolhidos como indicadores de desempenho ou resultado da ação estatal um conjunto de perguntas relacionadas às percepções dos burocratas em relação à eficácia da organização a que pertencem. Com isso, cumpriu-se também o requisito proposto por Andrews et al. (2011, p. 229), de que a medição do desempenho deve ser feita por meio de um "conjunto de medidas", em vez de apenas uma única que capture critérios relevantes. As questões do Survey selecionadas para compor o construto relacionada ao desempenho estatal foram as seguintes:

- $\mathrm{QH} 2$ - As políticas produzidas pela organização a qual pertenço atingiram os resultados esperados;

- QH1 - Atualmente, sua organização alcançou mais ou menos resultados, comparado a cinco anos atrás;

- QD4 - O desempenho da minha organização é bem-avaliado pela sociedade.

Como as demais questões, as respostas a essas 3 afirmações obedeceram à escala de Likert, variando de 1 a 5 , sendo 1 total discordância e 5, total concordância com os enunciados. A título de comparação e benchmark, essas questões tiveram como base o questionário do FEVS aplicado junto à burocracia estadunidense. 
A primeira questão busca mensurar a percepção do burocrata a respeito do alcance pela sua organização dos resultados esperados. A segunda, mensurar a percepção do servidor a respeito da eficácia de sua organização num período de tempo mais longo tempo, 5 anos, visando indicar avanços ou retrocessos na percepção ao longo do tempo. Por último, a percepção do respondente a respeito da avaliação que a sociedade faz do desempenho da organização à qual pertence foi selecionado como o terceiro indicador.

Reconhecemos o caráter genérico e subjetivo dessas questões. Mas, graças à falta de instrumentos claros, objetivos e incontestáveis dentro da própria administração pública federal brasileira a fim de averiguar o desempenho organizacional, fomos compelidos a recorrer a questões e respostas disponíveis no Survey para mensurar o construto em questão.

Na Tabela 1, abaixo, encontram-se as estatísticas descritivas para as questões analisadas.

\section{MÉTODO}

A modelagem de equações estruturais (MEE) é uma técnica estatística de análise multivariada de dados usada para examinar relações entre variáveis observáveis e construções latentes. Ela permite que se testem proposições teóricas sobre como as variáveis latentes estão formadas, as relações entre elas e a direcionalidade de tais relacionamentos, numa suposição entre causas e efeitos. Assim, a MEE permite mensurar os efeitos diretos, indiretos e totais entre os construtos, conforme previsto pela teoria (Hair, Black, Babin, Anderson \& Tatham, 2009). Para Codes (2008), além de a MEE se caracterizar pela capacidade de especificar, estimar e testar relações hipotéticas entre grupos de variáveis, ela propicia que as relações entre variáveis e construtos sejam traduzidas sob forma gráfica ou pictórica, o que possibilita uma representação clara da teoria e do modelo hipotetizado, o qual, neste trabalho, se baseou na construção de 5 construtos derivados das abordagens acerca do conceito de capacidade estatal discutidas na segunda seção e operacionalizadas por meio dos indicadores mensurados com base nas questões do questionário discutidas na seção anterior (Quadro 2).

Assim, a MEE foi utilizada como técnica confirmatória do modelo, testando hipóteses subjacentes às abordagens citadas, em especial se os indicadores estão relacionados com cada dimensão constitutiva do conceito e se estas estão correlacionadas entre si e com o desempenho percebido do Estado.

A Figura 2 mostra o modelo testado por meio do diagrama de caminhos. Retângulos representam variáveis observadas (indicadores ou perguntas do Survey). As variáveis latentes (ou construtos) são representadas graficamente com ovais. A linha reta que aponta de uma variável latente para as variáveis observadas indica o efeito hipotetizado da variável latente nas variáveis observadas. Como o foco da modelagem estrutural está na estimativa de relações entre construções latentes, as linhas retas mostram o efeito estimado de cada dimensão constitutiva do conceito sobre o construto desempenho, e a seta curva entre as dimensões constitutivas indica a relação entre elas.

Tabela 1

Estatísticas descritivas

\begin{tabular}{|c|c|c|c|c|c|c|c|}
\hline Construto & Questão & $\begin{array}{l}\text { Discordo } \\
\text { fortemente }\end{array}$ & Discordo & $\begin{array}{l}\text { Nem concordo } \\
\text { nem discordo }\end{array}$ & Concordo & $\begin{array}{l}\text { Concordo } \\
\text { fortemente }\end{array}$ & $\begin{array}{c}\text { Não sei/ } \\
\text { Não quero responder }\end{array}$ \\
\hline \multirow{6}{*}{ Profissionalização } & \multirow{2}{*}{ B1 } & 240 & 814 & 538 & 1037 & 252 & 23 \\
\hline & & $8,30 \%$ & $28,00 \%$ & $18,50 \%$ & $35,70 \%$ & $8,70 \%$ & $0,80 \%$ \\
\hline & \multirow{2}{*}{ B2 } & 562 & 990 & 597 & 576 & 112 & 67 \\
\hline & & $19,40 \%$ & $34,10 \%$ & $20,60 \%$ & $19,80 \%$ & $3,90 \%$ & $2,30 \%$ \\
\hline & \multirow{2}{*}{$\mathrm{B} 5 *$} & 56 & 117 & - & 578 & 2136 & 17 \\
\hline & & $1,90 \%$ & $4,00 \%$ & - & $19,90 \%$ & $73,60 \%$ & $0,60 \%$ \\
\hline \multirow{6}{*}{ Autonomia } & \multirow{2}{*}{$\mathrm{C} 1$} & 405 & 1101 & 640 & 612 & 124 & 22 \\
\hline & & $13,90 \%$ & $37,90 \%$ & $22,00 \%$ & $21,10 \%$ & $4,30 \%$ & $0,80 \%$ \\
\hline & \multirow{2}{*}{$\mathrm{C} 2$} & 268 & 640 & 584 & 1058 & 332 & 22 \\
\hline & & $9,20 \%$ & $22,00 \%$ & $20,10 \%$ & $36,40 \%$ & $11,40 \%$ & $0,80 \%$ \\
\hline & \multirow{2}{*}{ C3 } & 136 & 515 & 469 & 1343 & 426 & 15 \\
\hline & & $4,70 \%$ & $17,70 \%$ & $16,20 \%$ & $46,20 \%$ & $14,70 \%$ & $0,50 \%$ \\
\hline
\end{tabular}




\begin{tabular}{|c|c|c|c|c|c|c|c|}
\hline Construto & Questão & $\begin{array}{l}\text { Discordo } \\
\text { fortemente }\end{array}$ & Discordo & $\begin{array}{l}\text { Nem concordo } \\
\text { nem discordo }\end{array}$ & Concordo & $\begin{array}{l}\text { Concordo } \\
\text { fortemente }\end{array}$ & $\begin{array}{c}\text { Não sei/ } \\
\text { Não quero responder }\end{array}$ \\
\hline \multirow{6}{*}{ Recursos } & \multirow{2}{*}{ E1 } & 650 & 1102 & 319 & 683 & 121 & 29 \\
\hline & & $22,40 \%$ & $37,90 \%$ & $11,00 \%$ & $23,50 \%$ & $4,20 \%$ & $1,00 \%$ \\
\hline & \multirow{2}{*}{ E2 } & 805 & 1251 & 286 & 443 & 75 & 44 \\
\hline & & $27,70 \%$ & $43,10 \%$ & $9,80 \%$ & $15,30 \%$ & $2,60 \%$ & $1,50 \%$ \\
\hline & \multirow{2}{*}{ E3 } & 663 & 1159 & 305 & 645 & 103 & 29 \\
\hline & & $22,80 \%$ & $39,90 \%$ & $10,50 \%$ & $22,20 \%$ & $3,50 \%$ & $1,00 \%$ \\
\hline \multirow{6}{*}{ Habilidades } & \multirow{2}{*}{ G1 } & 99 & 548 & 477 & 1383 & 329 & 68 \\
\hline & & $3,40 \%$ & $18,90 \%$ & $16,40 \%$ & $47,60 \%$ & $11,30 \%$ & $2,30 \%$ \\
\hline & \multirow{2}{*}{ G3 } & 148 & 721 & 721 & 1005 & 228 & 81 \\
\hline & & $5,10 \%$ & $24,80 \%$ & $24,80 \%$ & $34,60 \%$ & $7,90 \%$ & $2,80 \%$ \\
\hline & \multirow{2}{*}{ G7 } & 151 & 582 & 632 & 1290 & 213 & 36 \\
\hline & & $5,20 \%$ & $20,00 \%$ & $21,80 \%$ & $44,40 \%$ & $7,30 \%$ & $1,20 \%$ \\
\hline \multirow{4}{*}{ Relacionamentos } & \multirow{2}{*}{ F8 } & 968 & 279 & 260 & 537 & 746 & 114 \\
\hline & & $33,30 \%$ & $9,60 \%$ & $9,00 \%$ & $18,50 \%$ & $25,70 \%$ & $3,90 \%$ \\
\hline & \multirow{2}{*}{ F10 } & 1624 & 391 & 287 & 304 & 150 & 148 \\
\hline & & $55,90 \%$ & $13,50 \%$ & $9,90 \%$ & $10,50 \%$ & $5,20 \%$ & $5,10 \%$ \\
\hline \multirow{6}{*}{ Desempenho } & \multirow{2}{*}{$\mathrm{H} 1$} & 180 & 418 & 451 & 1162 & 395 & 298 \\
\hline & & $6,20 \%$ & $14,40 \%$ & $15,50 \%$ & $40,00 \%$ & $13,60 \%$ & $10,30 \%$ \\
\hline & \multirow{2}{*}{$\mathrm{H} 2$} & 118 & 673 & 691 & 1101 & 185 & 136 \\
\hline & & $4,10 \%$ & $23,20 \%$ & $23,80 \%$ & $37,90 \%$ & $6,40 \%$ & $4,70 \%$ \\
\hline & \multirow{2}{*}{ D4 } & 169 & 653 & 669 & 999 & 309 & 105 \\
\hline & & $5,80 \%$ & $22,50 \%$ & $23,00 \%$ & $34,40 \%$ & $10,60 \%$ & $3,60 \%$ \\
\hline
\end{tabular}

Nota: $\left({ }^{*}\right)$ As alternativas para a QB5 foram: Não importante; Pouco importante; Importante; Muito importante; Não sei/Não quero responder.

Fonte: Elaborada pelos autores baseado em Pereira et al. (2019). 
Figura 2

Diagrama de caminhos

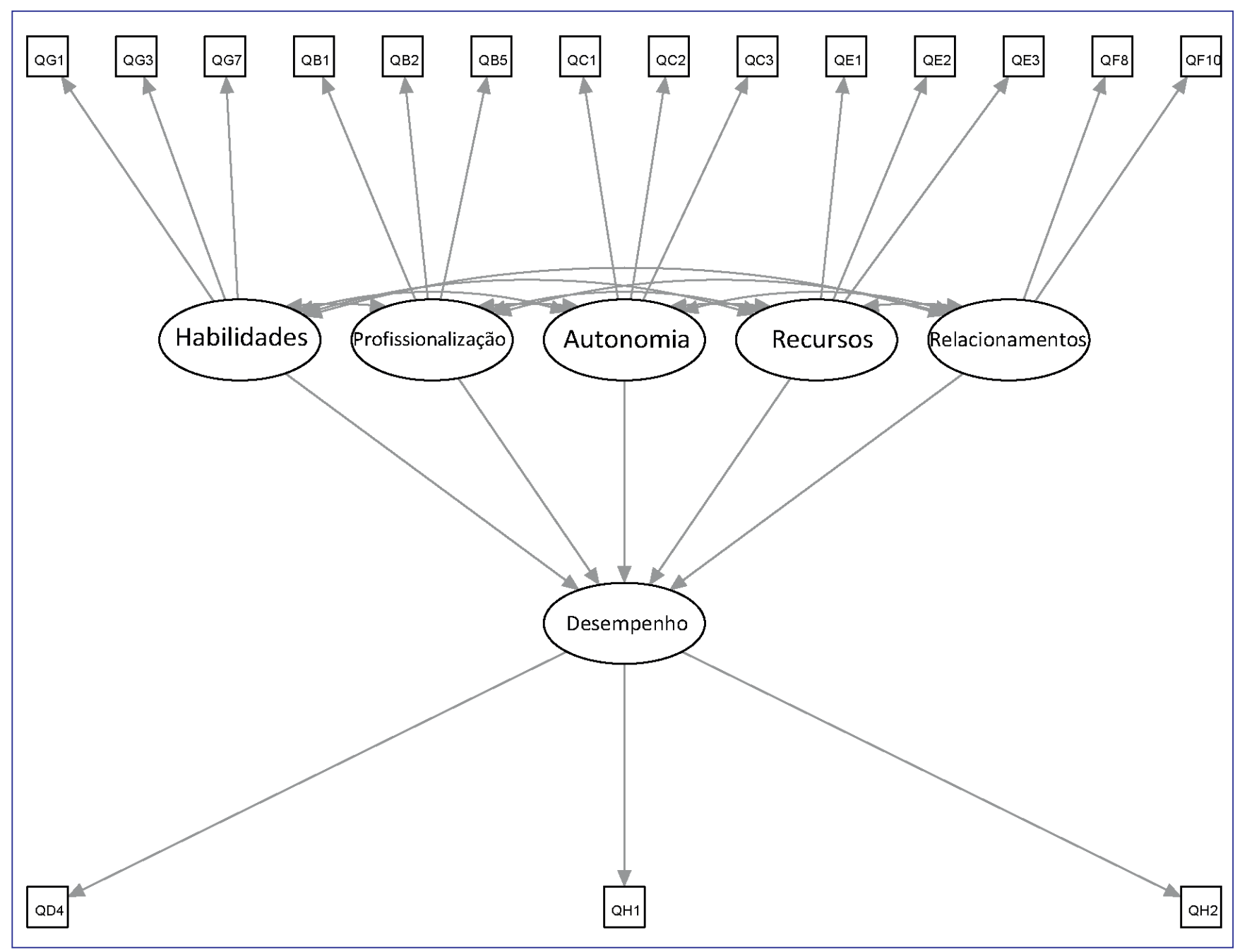

Fonte: Elaborada pelos autores.

\section{RESULTADOS}

O programa R (pacote Lavaan), com estimativa de máxima verossimilhança, foi utilizado na análise. $O$ modelo hipotetizado apresentou um bom ajuste para os dados (Meade, Johnson \& Braddy, 2008). Assim, não foram realizadas modificações post-hoc. O índice CFI (comparative fit index) indicou que os dados da amostra estão bem ajustados ao modelo $(0,93)$. O critério de corte para esse índice é $\mathrm{CFI} \geq 0,90$, evitando que modelos mal-especificados sejam aceitos (Hu \& Bentler, 1999). As saídas do programa $\mathrm{R}$ se encontram no anexo.

O RMSEA (root mean square error of approximation) foi de 0,052, estando nos limites indicados como um bom ajuste do modelo. De acordo com Hu e Bentler (1999), um ponto de corte do índice seria próximo a 0,06, com um limite de 0,07 (cf. também Steiger, 2007). Para Hooper, Coughlan e Mullen (2008), esse é o consenso entre os autores sobre o índice.

O outro índice de ajuste, o SRMR (standardised root mean square residual), que é a raiz quadrada da diferença entre os resíduos da amostra da matriz de covariância e o modelo de covariância hipotético, foi de 0,044 . Os valores desse índice variam de 0 a 1, com bons ajustes a menos de .05 (Byrne, 2013; Diamantopoulos \& Siguaw, 2000). Assim, o índice SRMR da análise indicou igualmente um bom ajuste do modelo. 


\section{Variáveis manifestas e latentes}

Em relação a variáveis latentes ou construtos, pudemos corroborar as expectativas teóricas para maioria dos indicadores selecionados. Com algumas exceções, discutidas abaixo, as cargas fatoriais dos indicadores selecionados foram altas $(>0,5)$ e estatisticamente significativas usando o valor de $p<0,05$ como ponto de corte.

No construto "profissionalização", encontramos uma carga fatorial baixa $(0,141)$ para a questão sobre o grau de importância da competência técnica para a nomeação de cargos comissionados. Conforme os respondentes, a competência técnica influencia pouco a nomeação para cargos de direção ou assessoramento superior na burocracia federal brasileira. Uma explicação para o resultado encontrado reside no fato de que os cargos em comissão na burocracia federal brasileira são de livre nomeação, ou seja, podem ser dados para pessoas de fora das carreiras do serviço público seguindo critérios políticos ou de relações pessoais. Como se sabe, a dinâmica de nomeações a cargos comissionados está intimamente ligada à estrutura institucional do presidencialismo de coalizão brasileiro (Lopez, 2015) - uma tipologia dessa relação pode ser encontrada em Batista e Lopez (2021).

Em relação ao construto "autonomia", todos os caminhos em direção aos indicadores selecionados foram estatisticamente significativos e caracterizados por altas cargas fatoriais $(>0,6)$, exceto para a questão relativa à concordância com a afirmação de que os burocratas teriam condições para tomar decisões próprias na realização de seus trabalhos, que apresentou carga fatorial baixa $(<0,5)$. Tal resultado pode indicar que, em termos gerais, há uma limitação à autonomia dos burocratas para execução de seus mandatos, via microgerenciamento ou interferências políticas na execução das tarefas a eles delegadas.

Por sua vez, relações estatisticamente significativas e altas cargas fatoriais foram encontradas nos caminhos entre os construtos "habilidades" e "recursos", e seus respectivos indicadores. Esse resultado indica que as variáveis observadas são explicadas pelos construtos, uma vez que, quanto maior a carga fatorial, mais o construto explica a variabilidade nos indicadores.

Em relação ao construto "relacionamentos", ambas as questões selecionadas apresentaram caminhos estatisticamente significativos, e o indicador da frequência da interação dos burocratas com as empresas privadas exibiu uma carga fatorial baixa $(<0,5)$ em comparação com o indicador relativo à frequência de interação com a sociedade civil $(>0,7)$. Isso pode indicar que, no geral, os burocratas interagem menos com as empresas privadas do que com a sociedade civil. Contudo, isso pode variar de acordo com o setor de política pública ou tipo de organização.

Por fim, mas não menos importante, os caminhos entre o construto "desempenho" e os 3 indicadores selecionados foram estatisticamente significativos e apresentaram altas cargas fatoriais $(>0,5)$, à exceção da pergunta relativa à percepção dos respondentes sobre a avaliação da sociedade a respeito do desempenho de suas respectivas organizações, que apresentou carga fatorial baixa $(<0,5)$. Isso indica que, segundo a percepção dos burocratas, a avaliação que a sociedade faz do desempenho de suas respectivas organizações têm pouca relação com o que é percebido por eles.

\section{Relações entre os construtos}

Usando o valor de $\mathrm{p}<0,05$ como ponto de corte para significância estatística, a Tabela 2 mostra uma forte correlação positiva $(>0,50)$ e significativa entre os construtos "profissionalização" e "autonomia", e entre "habilidades" e "profissionalização". Isso indica que, no caso brasileiro, profissionalização e autonomia burocrática andam juntas, assim como a seleção dos servidores está relacionada às habilidades técnicas deles.

Evocando o conceito de autonomia inserida (Evans, 1995), a covariâncias entre os construtos "autonomia" e "relacionamentos" foi significativa, porém fraca $(<0,50)$. Do mesmo modo, encontramos covariâncias fracas, mas estatisticamente significativas, entre os demais construtos - à exceção de "autonomia" e "recursos", e entre "recursos" e "relacionamentos", que não foram estatisticamente significativas $(p>0,10)$. Ressaltamos, contudo, que tais relações não eram esperadas teoricamente. 
Tabela 2

Covariância entre os construtos

\begin{tabular}{lcccc}
\hline & Estimate & Std.Err & z-value & $\mathrm{P}(>|z|)$ \\
\hline Habilidades & & & & \\
Profissionalização & 0,569 & 0,031 & 18,255 & 0,000 \\
Autonomia & 0,420 & 0,032 & 13,332 & 0,000 \\
Recursos & 0,089 & 0,038 & 2,331 & 0,020 \\
Relacionamentos & 0,178 & 0,040 & 4,479 & 0,000 \\
\hline Profissionalização & & & & 0,000 \\
Autonomia & 0,730 & 0,026 & 27,922 & 0,000 \\
Recursos & 0,208 & 0,041 & 5,046 & 0,021 \\
Relacionamentos & 0,094 & 0,041 & 2,315 & 0,307 \\
\hline Autonomia & & & & 0,000 \\
Recursos & 0,040 & 0,039 & 1,022 & \\
Relacionamentos & 0,218 & 0,042 & 5,219 & 0,100 \\
\hline Recursos & & 0,037 & $-1,645$ & \\
Relacionamentos & $-0,062$ & & & \\
\hline
\end{tabular}

Fonte: Elaborada pelos autores.

No que concerne aos efeitos hipotetizados de cada dimensão constitutiva do conceito e a variável latente "desempenho", encontramos efeitos positivos e estatisticamente significativos para os coeficientes de caminhos das dimensões "profissionalização" e "habilidades", vide a Tabela 3. O coeficiente de caminho entre "profissionalização" e "desempenho" foi o único maior que 0,50. Tais achados corroboram a expectativa teórica de que a profissionalização e a expertise burocráticas são variáveis preditoras do desempenho estatal.

Figura 3

Profissionalização como variável mediadora entre autonomia e desempenho

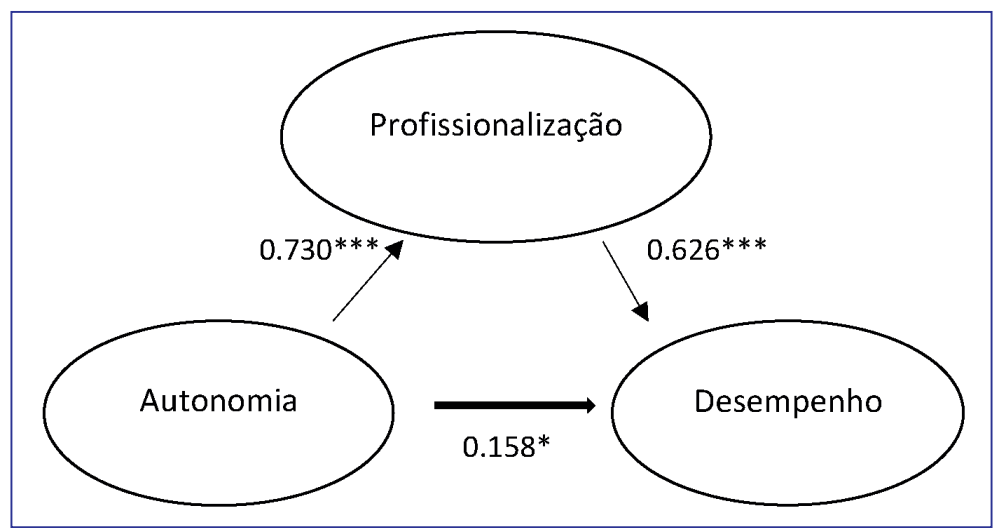

Nota: ${ }^{* * *} p<0,001 ; * * p<0,05 ; * p<0,10$

Fonte: Elaborada pelos autores. 
Já o coeficiente de caminho entre as dimensões "autonomia" e "desempenho" foi significativo apenas para $p<0,10$. Contudo, dado que a covariância entre profissionalização e autonomia é forte $(0,730)$ e estatisticamente significativa $(p<0,001)$, os efeitos da autonomia sobre o desempenho são mediados pela profissionalização. Ou seja, profissionalização se encaixa no caminho entre as variáveis "autonomia" e "desempenho", sendo que a profissionalização está significativamente associada ao desempenho e a autonomia influencia significativamente a profissionalização. Assim, tal variável medeia o efeito da autonomia burocrática sobre o desempenho estatal (Holmebeck, 1997), conforme ilustrado na Figura 3. Contrariando a expectativa teórica, não foram encontrados efeitos significativos do construto "recursos" sobre "desempenho".

Tabela 3

Correlação entre desempenho e dimensões do conceito

\begin{tabular}{lcccc}
\hline & Estimate & STD.ERR & z-value & $P(>|z|)$ \\
\hline Desempenho & & & & \\
Autonomia & 0,158 & 0,089 & 1,775 & 0,076 \\
Profissionalização & 0,626 & 0,118 & 5,288 & 0,000 \\
Relacionamentos & $-0,022$ & 0,043 & $-0,524$ & 0,600 \\
Recursos & $-0,037$ & 0,044 & $-0,830$ & 0,406 \\
Habilidades & 0,225 & 0,064 & 3,514 & 0,000 \\
\hline
\end{tabular}

Fonte: Elaborada pelos autores.

\section{CONCLUSÕES}

Este artigo especificou e testou um modelo teórico derivado de abordagens da literatura acerca do conceito de capacidade estatal. Para isso, foram analisados, por meio da técnica de modelagem de equações estruturais, dados coletados diretamente com a burocracia federal via questionário. Pôde-se, assim, mensurar as dimensões constitutivas do conceito, examinar as relações entre elas e verificar seus efeitos sobre o desempenho percebido do Estado.

Com as formulações de Fukuyama (2013) e Evans (1995), operacionalizamos os construtos de profissionalização e autonomia burocrática, assim como verificamos o nível de interação dos servidores federais com atores não estatais. Com base em Wu et al. (2018), aferimos as habilidades individuais e os recursos organizacionais nas organizações públicas federais.

A MEE foi usada como técnica confirmatória para testar o modelo teórico hipotetizado e estimar os efeitos das dimensões constitutivas da capacidade estatal sobre o desempenho do Estado. Ao proceder dessa maneira, evitamos os problemas de raciocínio circular presentes nas tentativas de mensurar o conceito.

Nossos achados indicam que os critérios weberianos de burocracia ainda são uma referência para estudos sobre a capacidade do Estado, uma vez que a profissionalização e as habilidades (ou expertise) dos burocratas estão diretamente relacionadas com o desempenho percebido das organizações estatais. No que concerne à autonomia burocrática, os achados indicam que os efeitos desta sobre o desempenho são mediados pela profissionalização. Ou seja, autonomia e profissionalização andam juntas, e quanto maior o nível de profissionalização burocrática, maior o efeito da autonomia sobre o desempenho organizacional.

Os achados também corroboraram a literatura ao afirmar que a competência técnica influencia pouco a nomeação para cargos de direção ou assessoramento superior na burocracia federal brasileira graças à existência de cargos de livre nomeação e à estrutura institucional do presidencialismo de coalizão. Disso também decorre a percepção dos burocratas sobre a limitação da autonomia para execução de seus mandatos, via microgerenciamento ou interferências políticas na execução de suas tarefas.

Apesar disso, ao contrário das previsões teóricas, não encontramos efeitos diretos significativos para o desempenho estatal dos relacionamentos da burocracia com os atores não estatais ou dos recursos organizacionais. Talvez os efeitos dessas variáveis sejam significativos apenas para determinadas organizações ou áreas de política pública. No entanto, não foi objetivo deste trabalho testar tal hipótese, merecendo aprofundamento em etapas posteriores da pesquisa. 
Uma burocracia competente e dotada de autonomia é apenas um potencial que precisa ser devidamente explorado pelo poder político para que se concretize em eficácia. Como afirmam Centeno et al. (2017), o fato de o Estado deter capacidade não quer dizer que necessariamente a utilizará, de modo que capacidade é uma espécie de potencial que deve ser acionado politicamente para ser concretizada.

As limitações deste trabalho apontam para a necessidade de pesquisas adicionais sobre o tema. Primeiro, a natureza transversal dos dados implica dificuldades em fornecer conclusões robustas sobre as relações causais entre as dimensões conceituais e o desempenho do Estado. Portanto, são necessárias pesquisas baseadas em dados longitudinais para robustecer tal tipo de inferência. Segundo, embora ciente das limitações dos Surveys para a mensuração da capacidade estatal e a medição do desempenho das ações do Estado, seria desejável usar indicadores objetivos para dar mais consistência aos achados, bem como combinar e complementar a análise com dados e análises qualitativas, numa abordagem multimétodo. Por fim, como a capacidade estatal não é um atributo fixo, mais pesquisas são necessárias para verificar e comparar tais variações entre setores de política e organizações públicas e entre governos subnacionais. 


\section{REFERÊNCIAS}

Andrews, R., Boyne, G., \& Walker, R. M. (2011). The impact of management on administrative and survey measures of organizational performance. Public Management Review, 13(2), 227-255.

Batista, M., \& Lopez, F. (2021). Ministerial Typology and Political Appointments: Where and How Do Presidents Politicize the Bureaucracy? Brazilian Political Science Review, 15(1), e0004.

Beaujean, A. A. (2014). Latent variable modeling using R: A step-bystep guide. London, UK: Routledge.

Bersch, K., Praça, S., \& Taylor, M. M. (2017). Bureaucratic capacity and political autonomy within national states: mapping the archipelago of excellence in Brazil. In M. A. Centeno, A. K. Kohli, D. J. Yashar, \& D. Mistree (Eds), States in the developing world (pp.157-183). Cambridge, UK: Cambridge University Press.

Boittin M., Distelhorst, G., \& Fukuyama, F. (2016). Reassessing the Quality of Government in China (Osgoode Legal Studies Research Paper Series, 197). Toronto, Canada: Osgoode Hall Law School of York University.

Bollen, K. A. (1989). A new incremental fit index for general structural equation models. Sociological methods \& research, 17(3), 303-316.

Byrne, B. M. (2013). Structural equation modeling with Mplus: Basic concepts, applications, and programming. London, UK: Routledge.

Carpenter, D. (2020). The forging of bureaucratic autonomy: Reputations, networks, and policy innovation in executive agencies, 1862-1928 (Vol. 173). Princeton, NJ: Princeton University Press.

Cingolani, L. (2018). The role of state capacity in development studies. Journal of Development Perspectives, 2(1-2), 88-114.

Codes, A. L. (2008). A trajetória do pensamento científico sobre pobreza: em direção a uma visão complexa (Texto para Discussão, no. 1332). Brasília, DF: Instituto de Pesquisa Econômica Aplicada.

Diamantopoulos, A., Siguaw, J. A., \& Siguaw, J. A. (2000). Introducing LISREL: A guide for the uninitiated. Thousand Oaks, CA: Sage.

Enriquez, E., \& Centeno, M. A. (2012). State capacity: utilization, durability, and the role of wealth vs. history. International and multidisciplinary journal of social sciences, 1(2), 130-162.

Evans, P. B. (1995). Embedded autonomy: States and industrial transformation. Princeton, NJ: Princeton University Press.

Evans, P. B. (2009). Constructing the 21st-century developmental state-potentialities and pitfalls. New Agenda: South African Journal of Social and Economic Policy, 36, 6-13.

Evans, P. B., \& Rauch, J. E. (1999). Bureaucracy and growth: A cross-national analysis of the effects of" Weberian" state structures on economic growth. American sociological review, 64(5), 748-765.

Fernandez, S., \& Moldogaziev, T. (2013). Employee empowerment, employee attitudes, and performance: Testing a causal model. Public Administration Review, 73(3), 490-506.

Fernandez, S., \& Moldogaziev, T. (2015). Employee empowerment and job satisfaction in the US Federal Bureaucracy: A self-determination theory perspective. The American review of public administration, 45(4), 375-401.
Fukuyama, F. (2013). What is governance?. Governance, 26(3), 347-368.

Gaitán, F., \& Boschi, R. R. (2016). Estado, Atores Predominates e Coalizões para o Desenvolvimento: Brasil e Argentina em Perspectiva Comparada. In A. A. Gomide, R. R. Boschi (Eds.), Capacidades Estatais em Países Emergentes: o Brasil em perspectiva comparada (pp. 473 506). Rio de Janeiro, RJ: Instituto de Pesquisa Econômica Aplicada.

Gingerich, D. W. (2013). Political institutions and party-directed corruption in South America: Stealing for the team. Cambridge, UK: Cambridge University Press.

Goertz, G. (2006). Social science concepts: A user's guide. Princeton, NJ: Princeton University Press.

Gomes, E. (2016). Relações Estado-Sociedade e Novas Capacidades Estatais para o Desenvolvimento entre os Países do BRICS: O Brasil em Perspectiva Comparada com a África do Sul e a Índia. In A. A. Gomide, R. R. Boschi (Eds.), Capacidades Estatais em Países Emergentes: o Brasil em perspectiva comparada (pp. 105-136). Rio de Janeiro, RJ: Instituto de Pesquisa Econômica Aplicada.

Grin, E. J., Nascimento, A. B., Abrucio, F. L., \& Fernandes, A. S. (2018). Sobre desconexões e hiatos: uma análise de capacidades estatais e finanças públicas em municípios brasileiros. Cadernos Gestão Pública e Cidadania, 23(76), 312-336.

Hair, J. F., Black, W. C., Babin, B. J., Anderson, R. E., \& Tatham, R. L. (2009). Análise multivariada de dados. Porto Alegre, RS: Bookman Editora.

Hanson, J. K., \& Sigman, R. (2019). State Capacity and World Bank Project Success. In Proceedings of the 2019 annual meeting of the American Political Science Association, Washington, DC.

Holmbeck, G. N. (1997). Toward terminological, conceptual, and statistical clarity in the study of mediators and moderators: examples from the child-clinical and pediatric psychology literatures. Journal of consulting and clinical psychology, 65(4), 599-610.

Hu, L. T., \& Bentler, P. M. (1999). Cutoff criteria for fit indexes in covariance structure analysis: Conventional criteria versus new alternatives. Structural equation modeling: a multidisciplinary journal, 6(1), 1-55.

Kocher, M. A. (2010, julho). State capacity as a conceptual variable. Yale Journal of International Affairs, 5(2), 137-145.

Lavalle, A. G., Carlos, E., Dowbor, M., \& Szwako, J. (2019). Movimentos sociais, institucionalização e domínios de agência. In A. G. Lavalle, E. Carlos, M. Dowbor \& J. Szwako (Eds.), Movimentos sociais e institucionalização: políticas sociais, raça e gênero no Brasil pós-transição (pp. 21-88). Rio de Janeiro, RJ: EdUERJ.

Lopez, F. G. O. (2015). Cargos de confiança no presidencialismo de coalizão brasileiro. Rio de Janeiro, RJ: Instituto de Pesquisa Econômica Aplicada.

March, J. G., \& Sutton, R. I. (1997). Crossroads-organizational performance as a dependent variable. Organization science, 8(6), 698-706. 
Marenco, A., Strohschoen, M. T. B., \& Joner, W. (2017). Capacidade estatal, burocracia e tributação nos municípios brasileiros. Revista de Sociologia e Política, 25(64), 3-21.

Meade, A. W., Johnson, E. C., \& Braddy, P. W. (2008). Power and sensitivity of alternative fit indices in tests of measurement invariance. Journal of applied psychology, 93(3), 568-592.

Painter, M., \& Pierre, J. (2005). Unpacking policy capacity: Issues and themes. In M. Painter, \& J. Pierre (Eds.), Challenges to state policy capacity (pp. 1-18). London, UK: Palgrave Macmillan.

Pereira, A. K., Machado, R. A., Cavalcante, P. L. C., Gomide, A. A., Bersch, K., Magalhães, A. G. ... Pires, R. R. C. (2019). Qualidade do governo e capacidade estatal (Relatório de Pesquisa). Brasília, DF: Instituto de Pesquisa Econômica Aplicada.

Pires, R. R. C., \& Gomide, A. D. Á. (2016). Governança e capacidades estatais: uma análise comparativa de programas federais. Revista de sociologia e política, 24(58), 121-143.

Ramesh, M., Howlett, M. P., \& Saguin, K. (2016). Measuring individuallevel analytical, managerial and political policy capacity: A survey instrument (Lee Kuan Yew School of Public Policy Research Paper, № 16-07). Suburb, Singapura: Lee Kuan Yew School of Public Policy at University of Singapore.

Roller, E. (2020). Political performance and state capacity. In D. Berg-Schlosser, B. Badie, L. Morlino (Eds.), The SAGE handbook of political science (Vol. 3, pp. 916-933). London, UK: SAGE Publications.

Sikkink, K. (1991). Ideas and institutions: developmentalism in Brazil and Argentina. Ithaca, NY: Cornell University Press.
Skocpol, T. (1985). Bringing the state back in: Strategies of analysis in current research. In P. B. Evans, D. Rueschemeyer, \& T. Skocpol (Eds.), Bringing the state back in (pp. 3-43). Cambridge, UK: Cambridge University Press.

Souza, C. (2016). Capacidade burocrática no Brasil e na Argentina: quando a política faz a diferença. In A. A. Gomide, \& R. R. Boschi (Eds.), Capacidades Estatais em Países Emergentes: o Brasil em perspectiva comparada (pp. 51-104). Rio de Janeiro, RJ: Instituto de Pesquisa Econômica Aplicada.

Souza, C., \& Fontanelli, F. (2020). Capacidade estatal e burocrática: sobre conceitos, dimensões e medidas. In J. Mello, V. M. Ribeiro, G. Lotta, A. Bonamino, \& C. P. Carvalho (Eds.), Implementação de políticas e atuação de gestores públicos (pp. 43-67). Brasília, DF: Instituto de Pesquisa Econômica Aplicada.

Steiger, J. H. (2007). Understanding the limitations of global fit assessment in structural equation modeling. Personality and Individual differences, 42(5), 893-898.

Teskey, G. (2011). State-building and development: getting beyond capacity. Commonwealth good governance, 12, 44-48.

Williams, M. J. (2018). Beyond state capacity: bureaucratic performance, policy implementation and reform. Journal of Institutional Economics, 17, 339-357.

Wu, X., Ramesh, M., \& Howlett, M. (2015). Policy capacity: A conceptual framework for understanding policy competences and capabilities. Policy and Society, 34(3-4), 165-171.

Alexandre de Ávila Gomide

ORCID: https://orcid.org/0000-0002-7979-8865

Doutor em Administração Pública e Governo pela Fundação Getulio Vargas de São Paulo; Membro da carreira de Planejamento e Pesquisa do Instituto de Pesquisa Econômica Aplicada (IPEA); Professor permanente do mestrado profissional em Administração Pública do Instituto Brasileiro de Educação, Desenvolvimento e Pesquisa (IDP) e do mestrado profissional em Políticas Públicas e Desenvolvimento do Instituto de Pesquisa Econômica Aplicada (IPEA).E-mail: alexandre.gomide@ipea.gov.br

Raphael Amorim Machado

ORCID: https://orcid.org/0000-0001-7500-4485

Doutor em Ciência Política pela Universidade Estadual de Campinas (Unicamp); Pesquisador visitante na Diretoria de Estudos e Políticas do Estado, Instituições e Democracia do Instituto de Pesquisa Econômica Aplicada (IPEA); Professor substituto do Instituto de Ciência Política da Universidade de Brasília (UnB). E-mail: raphaelmachado2@gmail.com

Pedro Melo Albuquerque

ORCID: https://orcid.org/0000-0002-1415-716X

Doutor em Administração, Finanças e Métodos quantitativos pela Universidade de Brasília (UnB); Professor adjunto no Departamento de Administração da Universidade de Brasília (UnB) e coordenador do Laboratório de Aprendizado de Máquina em Finanças e Organizações - LAMFO (lamfo.unb.br) E-mail: pedroa@unb.br 